\title{
Anticancer Drugs Induced Severe Adverse Cutaneous Drug Reactions: An Updated Review on the Risks Associated with Anticancer Targeted Therapy or Immunotherapies
}

\author{
Chau Yee $N g \mathbb{D}^{1,2,3,4}$ Chun-Bing Chen $\mathbb{D}^{1,2,3,4}$ Ming-Ying $W u^{1,2,3}$ Jennifer $W u,{ }^{1,2,3}$ \\ Chih-Hsun Yang, ${ }^{1,2,3,4}$ Rosaline Chung-Yee Hui,, ${ }^{1,2,3,4}$ Ya-Ching Chang, ${ }^{1,2,3,4}$ \\ and Chun-Wei Lu iD 1,2,3,4 \\ ${ }^{1}$ Department of Dermatology, College of Medicine, Chang Gung Memorial Hospital, Keelung, Linkou, Taipei, Taiwan \\ ${ }^{2}$ Drug Hypersensitivity Clinical and Research Center, Chang Gung Memorial Hospital, Taipei, Taiwan \\ ${ }^{3}$ School of Medicine, College of Medicine, Chang Gung University, Taoyuan, Taiwan \\ ${ }^{4}$ Graduate Institute of Clinical Medical Sciences, Chang Gung University, Taoyuan, Taiwan \\ Correspondence should be addressed to Chun-Wei Lu; c.wei.lu@gmail.com
}

Received 31 August 2017; Revised 7 November 2017; Accepted 8 November 2017; Published 17 January 2018

Academic Editor: Riichiro Abe

Copyright (C) 2018 Chau Yee Ng et al. This is an open access article distributed under the Creative Commons Attribution License, which permits unrestricted use, distribution, and reproduction in any medium, provided the original work is properly cited.

\begin{abstract}
Cutaneous adverse drug reactions are commonly seen in patients with anticancer drug treatment. Anticancer drugs, including chemotherapy, target therapy, and recent immunotherapy causing skin reactions ranging from mild skin rash to life-threatening severe cutaneous adverse reactions (SCARs), such as Stevens-Johnson syndrome (SJS) and toxic epidermal necrosis (TEN) with increase morbidity and mortality while they are receiving cancer treatments, have been proposed to be a result of direct skin toxicity or drug hypersensitivity reactions (these are proposed mechanism, not definite). Differentiating SCARs from other more commonly seen reactions with a better outcome help prevent discontinuation of therapy and inappropriate use of systemic immunosuppressants for presumable allergic reactions, of which will affect the clinical outcome. In this article, we have reviewed published articles from 1950 to August 2017 for SJS/TEN associated with anticancer drugs, including chemotherapy, targeted therapy, and immunotherapy. We aimed to provide an overview of SJS/TEN associated with anticancer drugs to increase clinician recognition and accelerate future studies on the pathomechanism and managements.
\end{abstract}

\section{Introduction}

The advancement in cancer detection and development of anticancer drug therapy has led to increased incidence of cutaneous adverse reactions following anticancer drug therapy. Conventional chemotherapy and targeted or immunotherapy that are thought to be well tolerated and may cause various cutaneous adverse reactions ranging from nonlifethreatening skin toxicities such as paronychia, acneiform eruption, and alopecia to life-threatening severe cutaneous adverse reactions (SCARs) such as Stevens-Johnson syndrome (SJS) and toxic epidermal necrolysis (TEN). These drug eruptions are thought to be immunologically mediated reactions that are termed type $B$ adverse reaction [1].
However, the pathomechanism of SCARs reactions in anticancer drugs including chemotherapy, targeted therapy, and immunotherapy is poorly understood and the literatures were still limited.

SJS/TEN are a spectrum of fatal mucocutaneous adverse reactions characterized by rapidly progressing purpuric atypical target-like rashes with blisters, cutaneous sloughing, and mucosal involvement. SJS and TEN are differentiated by the degree of skin detachment: SJS involves less than $10 \%$ body surface area skin detachment, TEN more than $30 \%$, while SJS/TEN overlap involves body surface area of $10-30 \%[1,2]$. Despite their rare occurrence, the overall mortality was generally high in accordance with the body surface involve, ranging from $10 \%$ for SJS to approximately 
TABle 1: Anticancer chemotherapy-related severe cutaneous adverse drug reactions from the English literature (year: 1950-2017).

\begin{tabular}{|c|c|c|c|c|c|c|c|c|}
\hline Drug class & Drug & Pharmacology & References & Total $(n)$ & Mortality & SJS & SJS/TEN & TEN \\
\hline \multirow{5}{*}{ Alkylating agents } & Treosulfan & Alkysufonates & {$[6]$} & 1 & 1 & 0 & 0 & 1 \\
\hline & Chlorambucil & Mustard gas derivatives & {$[7,8]$} & 2 & 0 & 0 & 0 & 2 \\
\hline & Mechlorethamine (topical) & Nitrogen mustard & [9] & 1 & 0 & 1 & 0 & 0 \\
\hline & Temozolomide & Hydrazines and triazines & [10] & 1 & 0 & 0 & 1 & 0 \\
\hline & Procarbazine & Hydrazines and triazines & {$[11-13]$} & 3 & 0 & 0 & 0 & 3 \\
\hline \multirow{3}{*}{ Plant alkaloids } & Paclitaxel & Taxanes & {$[14]$} & 1 & 0 & 1 & 0 & 0 \\
\hline & Docetaxel & Taxanes & [15-19] & 5 & 2 & 3 & 0 & 2 \\
\hline & Etoposide & Podophyllotoxins & {$[20]$} & 1 & 0 & 1 & 0 & 0 \\
\hline Anthracyclines & Doxorubicin & & [21] & 1 & 1 & 0 & 0 & 1 \\
\hline \multirow{9}{*}{ Antimetabolites } & Methotrexate & Folic acid antagonists & {$[22-26]$} & 5 & 2 & 2 & 0 & 3 \\
\hline & Cytarabine & Pyrimidine antagonist & {$[27,28]$} & 2 & 2 & 0 & 0 & 2 \\
\hline & Fludarabine & Adenosine deaminase inhibitor & [29] & 1 & 1 & 1 & 0 & 0 \\
\hline & Gemcitabine & Pyrimidine antagonist & [30-32] & 3 & 0 & 2 & 1 & 0 \\
\hline & Capecitabine & Pyrimidine antagonist & [33] & 1 & 0 & 1 & 0 & 0 \\
\hline & Cladribine & Purine antagonist & {$[34,35]$} & 2 & NA & 1 & 0 & 1 \\
\hline & 6-Mercaptopurine & Purine antagonist & {$[36]$} & 1 & NA & 0 & 0 & 1 \\
\hline & \multicolumn{2}{|c|}{ TS-1 (tegafur-gimeracil-oteracil potassium) } & {$[37,38]$} & 2 & 0 & 1 & 0 & 1 \\
\hline & Pemetrexed & Multitarget antifolate & {$[39,40]$} & 2 & 0 & 0 & 0 & 2 \\
\hline \multirow{3}{*}{$\begin{array}{l}\text { Antitumor } \\
\text { antibiotics }\end{array}$} & Bleomycin & & {$[41,42]$} & 2 & 1 & 0 & 0 & 2 \\
\hline & Peplomycin & & {$[43]$} & 1 & 0 & 1 & 0 & 0 \\
\hline & Mithramycin & & {$[44,45]$} & 2 & 0 & 0 & 0 & 2 \\
\hline \multirow{4}{*}{ Miscellaneous } & Lenalidomide & & [46-48] & 14 & 2 & 12 & 1 & 1 \\
\hline & Thalidomide & & [49-53] & 5 & 1 & 1 & 0 & 4 \\
\hline & Asparaginase & & {$[54]$} & 1 & 0 & 0 & 0 & 1 \\
\hline & & & Total & 60 & 13 & 28 & 3 & 29 \\
\hline
\end{tabular}

NA: not available.

$50 \%$ for TEN, and can cause irreversible sequelae to the eyes, skin, and lungs [2-5]. Hence, increased recognition and improved management are of paramount importance, especially at early stages. Furthermore, in clinical practice, the conjectural association of anticancer drugs with SCAR event may lead to alterations in therapy, affects clinical outcome, and may cause physician and patient distress. This review aimed to provide an overview of the current evidence of anticancer drug-related SCARs to assist clinicians in early recognition and management.

To synthesize current literature, relevant English literatures were identified through searches of PubMed, EMBASE, Web of Science, SCOPUS, and OVID from 1950 to August 2017 using the terms Stevens-Johnson syndrome, toxic epidermal necrolysis, cancer drug therapy, and target therapy drugs. We did not constrain our research on publication types but limited the search only in indexed, peer-reviewed journals so as to ensure quality publications. Primary case reports, case series, reports from clinical trials, or as part of postmarketing surveillance were included. Histopathologic diagnosis of SJS/TEN was not required for the inclusion criteria. Clinical course, type of anticancer drugs, and mortality were analyzed and summarized according to the respective anticancer drug classifications of chemotherapy [6-54] (Table 1), targeted therapy [55-80]
(Table 2), and immunotherapy [81-87] (Table 3). Cases with multiple concomitant medications used during the same period of time and/or with questionable diagnosis were excluded.

\section{Chemotherapy}

Chemotherapy is the most widely used anticancer drug in oncology field. The administration of chemotherapy may lead to many cutaneous findings, ranging from allergic reactions to infectious complications caused by disrupted immunity. From the search of peer-review articles, a total of 60 reports of SJS/TEN associated with 23 chemotherapeutic anticancer drugs were identified [6-54] (Table 1). The most common drugs to cause chemotherapy-induced SJS/TEN are lenalidomide $(n=14 ; \mathrm{SJS}=12, \mathrm{SJS} / \mathrm{TEN}=1$, and TEN $=1)$, methotrexate $(n=5 ; \mathrm{SJS}=2$ and $\mathrm{TEN}=3)$, docetaxel $(n=4 ; \mathrm{SJS}=3$ and $\mathrm{TEN}=1)$, and thalidomide $(n=5 ;$ SJS $=1$ and TEN $=4)$. Most patients were exposed to drugs either concomitantly or within 8 weeks of the anticancer agent. Although there were a few cases with exceedingly short duration of onset with questionable diagnosis $[28,31]$, the report descriptions and causality indicators (course of treatment, duration and timing between exposure and event, blood levels, etc.) were not consistently 
TABLE 2: Anticancer targeted therapy-related severe cutaneous adverse drug reactions from the English literature (year: 1950-2017).

\begin{tabular}{|c|c|c|c|c|c|c|c|c|}
\hline Drug class & Drug & Pharmacology & References & Total $(n)$ & Mortality & SJS & SJS/TEN & TEN \\
\hline \multirow{6}{*}{ EGFR inhibitor } & Afatinib & Monoclonal antibody to EGFR & {$[55,56]$} & 2 & 0 & 2 & 0 & 0 \\
\hline & Cetuximab & Monoclonal antibody to EGFR & [57-59] & 4 & 1 & 1 & 1 & 2 \\
\hline & Erlotinib & TKI specific to EGFR & {$[60]$} & 1 & 0 & 1 & 0 & 0 \\
\hline & Gefitinib & TKI specific to EGFR & {$[61,62]$} & 2 & 1 & 0 & 0 & 2 \\
\hline & Panitumumab & Monoclonal antibody to EGFR & [122] & 1 & 0 & 1 & 0 & 0 \\
\hline & Vandetanib & Less specific multikinase inhibitors & {$[63]$} & 2 & 0 & 0 & 1 & 1 \\
\hline $\begin{array}{l}\text { KIT and BCR-ABL } \\
\text { inhibitors }\end{array}$ & Imatinib & KIT, BCR-ABL, PDGFR & [64-72] & 11 & 1 & 11 & 0 & 0 \\
\hline Antiangiogenic agents & Sorafenib & $\begin{array}{c}\text { Nonselective antiangiogenesis } \\
\text { multikinase agents }\end{array}$ & {$[73-76]$} & 3 & 0 & 2 & 0 & 1 \\
\hline Proteasome & Bortezomib & & {$[77]$} & 2 & 1 & 1 & 0 & 1 \\
\hline CD30 & Brentuximab vedotin & CD30 & {$[78]$} & 2 & 0 & 1 & 0 & 1 \\
\hline CD20 & Rituximab & Monoclonal antibody to CD20 & {$[79]$} & 5 & 2 & 2 & 2 & 1 \\
\hline \multirow[t]{2}{*}{ BRAF inhibitors } & Vemurafenib & A/B/C-Raf and B-Raf (V600E) & {$[80]$} & 7 & 1 & 1 & 0 & 6 \\
\hline & & & Total & 42 & 7 & 23 & 4 & 15 \\
\hline
\end{tabular}

TABle 3: Anticancer immune therapy-related adverse drug reactions from the English literature (year: 1950-2017).

\begin{tabular}{|c|c|c|c|c|c|c|c|c|}
\hline Drug class & Drug & Pharmacology & References & Total & Mortality & SJS & SJS/TEN & TEN \\
\hline \multirow{6}{*}{ Immunomodulators } & Aldesleukin & Recombinant interleukin-2 & {$[81,82]$} & 2 & 1 & 0 & 0 & 2 \\
\hline & Ipilimumab & CTLA-4 inhibitors & {$[83]$} & 1 & 0 & 1 & 0 & 0 \\
\hline & Nivolumab & PD-1 inhibitors & {$[84,85]$} & 2 & 1 & 0 & 0 & 2 \\
\hline & Pembrolizumab & PD-1 inhibitors & {$[86,114,116]$} & 4 & 0 & 4 & 0 & 0 \\
\hline & Denileukin & $\begin{array}{l}\text { Recombinant interleukin-2 and } \\
\text { diphtheria toxin }\end{array}$ & {$[87]$} & & 1 & 0 & 0 & 1 \\
\hline & & & Total & 9 & 3 & 5 & 0 & 5 \\
\hline
\end{tabular}

reported in these articles. Some articles enclose pictures that are not very suggestive of SJS/TEN but of an alternative diagnosis, including erythema multiforme, GVHD, and toxic erythema of chemotherapy. For instance, methotrexate-induced epidermal necrosis is a distinct entity that closely mimics SJS/TEN but exhibits distinct clinicopathological features from SJS/TEN [88]. Many of the reported articles did not obtain skin biopsy for pathology examination and hence, it is difficult to draw to a definitive diagnosis of SJS/TEN. Another clinical mimic of SJS/TEN associated with chemotherapy is toxic erythema of chemotherapy (TEC), characterized by painful erythematous eruptions with edema and/or blisters which involves the acral part, intertriginous areas, pressure points, and less often ears, knees, and elbows $[89,90]$. TEC is a toxic phenomenon with minimal inflammatory infiltrates despite the dramatic clinical appearance, hence studies have hypothesized that the erythema is secondary to keratinocyte damage with release of cytokines leading to vasodilation $[90,91]$. Most cases involve the use of either antimetabolites or alkylating agents that interferes RNA or DNA synthesis, including methotrexate, cytarabine, 5-fluorouracil, and mercaptopurine. By contrast, SJS/TEN is an immune-driven type 4 allergic reaction, where cytotoxic T lymphocytes and natural killer cells are activated. Clinical recognition and differentiation of SIS/TEN from toxic erythema are of importance because it helps prevent the inappropriate use of systemic immunosuppressants for presumed allergic reactions, precludes subsequent dosing, and affects the patient's clinical outcome.

\section{Targeted Anticancer Therapy}

From the literature review, a roster of 42 reports of SJS $(n=23)$, SJS/TEN $(n=4)$, or TEN $(n=15)$, associated with 12 targeted anticancer drugs, were identified, including EGFR inhibitors (afatinib, cetuximab, erlotinib, gefitinib, panitumumab, and vandetanib), MKI (imatinib, regorafenib, and sorafenib), recombinant IL-2 (aldesleukin), proteasome (bortezomib), anti-CD20 (rituximab), anti-CD30 (brentuximab vedotin), and BRAF inhibitor (vemurafenib) (Table 2). The most common drugs to cause SJS/TEN reported are imatinib $(n=11)$, EGFR inhibitors $(n=10)$, and vemurafenib $(n=7)$. The response of cancer control is hard to analyze because it was not fully mentioned in the reports. All cases were treated with immunosuppressant, including steroid, IVIG, and there was one TEN case with promising outcome after etanercept (anti-TNF $\alpha$ ) treatment. In these reports, nine patients underwent drug rechallenge test with recurrences, confirming the notoriety of exposed targeted anticancer drugs $[67-72,74,80]$. 
3.1. EGFR Inhibitors. EGFR inhibitors are approved as the drug for the treatment of non-small cell lung, colorectal, breast, pancreatic, head, and neck cancers with EGFR mutations [92]. The incidence of EGFR inhibitor-induced cutaneous adverse drug reactions (cADRs) is high (36\%$80 \%$ ) [93], of which most were papulopustular eruptions, xerosis, paronychia, mucositis, and photosensitivity [94]. In this article, we have identified 13 cases of SJS/TEN induced by EGFR inhibitors. Though rare, SJS/TEN should be distinguished from EGFR inhibitor-related mucositis, particularly when the patient present with constitutional symptoms and widespread atypical target spots with blisters that extend beyond mucosa to the skin. Crossreactivity between EGFR inhibitors was reported. It is hypothesized that the pathomechanism of SJS/TEN associated with EGFR inhibitors could be caused by to the irreversible inhibition of EGFR, of which hinders epidermal differentiation and reepithelialization and causing extensive erosions [95].

3.2. KIT and $B C R-A B L$ Inhibitors. Imatinib, a tyrosine kinase inhibitor, is the standard treatment in chronic myeloid leukemia and gastrointestinal stromal tumors (GIST) [96, 97]. In this article, imatinib accounts one of the most common causative targeted anticancer drug to induce SJS, with a roster of 12 cases. This must be differentiated from other more commonly seen cutaneous adverse effects of imatinib, maculopapular rashes, and facial edema [98], of which has a better prognosis and dose-dependent pharmacologic effect rather than hypersensitivity reaction [99]. For maculopapular rash/facial edema associated with imatinib, temporary discontinuation or dose reduction may be applied if the patient's cancer is susceptible to the drug. By contrast, reintroducing the culprit drug with a dose reduction is usually not suggested [100,101].

3.3. Multikinase Inhibitors. Multikinase inhibitors (sunitinib, sorafenib, pazopanib, and vandetanib) are small molecule inhibitors of the tyrosine kinase of the VEGF, and also differential binding capacities to other tyrosine kinases, including PDGFR, EGFR, KIT, RET, FLT-3, CSF-1R, and RAF [102]. They were approved for treatment of patients with renal cell cancer, gastrointestinal stromal tumors, and hepatocellular cancer. These drugs can cause hand-foot skin reaction, hair change, maculopapular eruptions, stomatitis, genital erosions, and bleeding $[103,104]$, especially in patients using sorafenib. These more common cutaneous toxicities are thought to be caused by direct VEGF inhibition, which result in vessel regression, and impact on vascular repair capacities [74]. Other research has also shown that Fas/FasL interaction mediates keratinocyte death in sunitinib-induced HFSR [75]. Recently, one recent study identified SLC22A20 (OAT6) as an uptake carrier of sorafenib and subsequently sorafenib enters the keratinocyte through OAT6 and then inhibits mitogen-activated protein kinase MAP3K7 (TAK1) leading to cytotoxicity and keratinocyte injury [76]. Interestingly, erythema multiforme, a spectrum of delayed type hypersensitivity, induced by sorafenib was around $19-25 \%$ in Japanese population, which is much higher than the Caucassian population [105]. This could imply a possible genetic role in the pathogenesis of adverse drug reactions. The different incidence of cutaneous adverse reactions among different ethnicities need to be further investigated.

3.4. BRAF Inhibitors. Vemurafenib is a selective inhibitor of BRAF-kinase approved for the treatment of metastatic melanoma with BRAF mutation. Skin toxicity, such as photosensitivity and maculopapular eruptions, and secondary skin malignancy (keratoacanthoma and squamous cell carcinoma) were estimated to affect more than $90 \%$ of patients $[106,107]$. One vemurafenib-TEN underwent a lymphocyte transformation test (LTT) assay to confirm the causality of vemurafenib and also show positive cross-reactivity for dabrafenib [108]. On the contrary, another case reported a successful switch from vemurafenib-induced cutaneous adverse reactions to dabrafenib [109]. Furthermore, cross-reactivity was also found between vemurafenib and sulfonamide antibiotics-sulfamethoxazole-based on LTT reports. These data suggested that there might be clinical cross-reactivity between BRAF inhibitors and sulfonamides. Predisposing factors to sulfonamide-related adverse cutaneous drug reactions could be implied in the pathomechanism studies of vemurafenib-associated SJS/TEN [108].

3.5. mTOR Inhibitors. Mammalian target of rapamycin (mTOR) inhibitors, such as sirolimus, everolimus, and temsirolimus, are emerging drugs, increasingly applied in oncology and in the prevention of rejection in patients receiving solid organ transplantation [110]. The most common cutaneous side effects are oral ulcers, acne-like eruptions, and morbilliform drug eruptions [111]. Oral ulcer is a very frequent $(72 \%)$ adverse reaction and is often recurrent and chronic following everolimus treatment in $25 \%$ of patients. The adverse event was found to be dose dependent [112].

Severe drug eruptions of life-threatening lingual angioedema after initiation of everolimus in heart transplant recipients have also been reported in a case series. In these patients, lingual edema occurs predominantly within the first weeks after initiation of everolimus therapy and disappears without recurrences in majority patients after adequate symptomatic treatment [113].

There were otherwise no SCAR (SJS/TEN, DRESS) event being reported in the literature.

\section{Immunotherapy}

Immunotherapy is the latest breakthrough in anticancer drug development with immunomodulatory therapeutic antibodies, targeting inhibitory receptors expressed by $\mathrm{T}$ cell as CTLA-4 and PD-1. They are used to treat advance stage cancer with metastasis or unresectable tumor such as melanoma and lung cancer. In this section, older immunotherapy such as interleukin-2 was also included in Table 3. These therapeutic options are most widely used in advanced and late cancer stages. From literature reviews, we have identified one ipilimumab-SIS, two nivolumabTEN, and four pembrolizumab-SJS. All of the patients were 
advanced melanoma patients, and the onset of epidermal necrolysis varies from 2.5 weeks to 3 months. In one case of pembrolizumab-associated SJS, concomitant phenytoin for epilepsy was used; hence, the exact culprit drug is hard to define. Two cases of pembrolizumab-SJS were being reported by Saw et al. [114]. Interestingly, there was a striking demarcation of epidermal detachment along the radiotherapy field aside from typical mucocutaneous findings of SJS. Such findings, although rarely, have also been reported in previous traditional culprit drugs and targeted therapy. A total of 3 cases were found with interleukin-2 immune therapy with 2 fatalities [81, 82, 87]. One of the authors suggested that IL-2 may increase patient's susceptibility to allergy of other medication [87]. An increased expression of PD-L1 in the epidermis by immunohistochemistry (IHC) was found, and they hypothesized that the use of anti-PD-1 therapy could provoke the expression of PD-L1 of keratinocytes and permit the activated $\mathrm{CD}^{+}$cytotoxic $\mathrm{T}$ cells to target keratinocytes leading to keratinocyte apoptosis [86]. PD-1 knockout mouse often exhibits symptoms related to adverse cutaneous reactions. It has been reported in a mouse model that PD-L1 expressed on keratinocytes presenting self-antigens regulates autoreactive $\mathrm{CD} 8^{+} \mathrm{T}$ cell activity and prevents the development of cutaneous autoimmune disease [115]. Goldinger et al. had demonstrated that the gene expression analysis of TEN-like lesional skin from antiPD-1-treated patients revealed an upregulation of major inflammatory chemokines, such as CXCL9, CXCL10, and CXCL11, of cytotoxic mediators such as PRF1 and GZMB and proapoptotic FASLG and upregulation of PD-L1 [116]. These gene expression profiles resembling SJS/TEN suggest that $\mathrm{PD}-1 / \mathrm{PD}-\mathrm{L} 1$ interaction is required to preserve epidermal integrity during inflammatory skin reactions. Interestingly, there was a case with preceding nivolumab treatment followed by vemurafenib who developed TEN [117]. The authors suggest that nivolumab predispose patients to drug hypersensitivity reactions through activation of $\mathrm{CD}^{+}$cells $[84,85]$.

In spite of being uncommon, SJS/TEN are severe lifethreatening cutaneous diseases that should be concerned in patients treated with anticancer drugs. The typical presentation and diagnosis often require proper drug exposure documentation, photography, and skin biopsies. Currently, there are many different classifications and models with detail and validated diagnostic criteria to assist clinical diagnosis and can help predict patients' mortality [118, 119]. Standard reporting method is important for subsequent investigation and analysis of these rare events. In addition, diagnosis of culprit drug is often challenging, the drug notoriety scoring systems including ALDEN score, Naronjo score and in vitro test with lymphocyte transformation test (LTT) are useful tests for the diagnosis of drug hypersensitivity and cross-reactivity and helped to better understand these reactions $[120,121]$. Current evidence on the pathomechanism of this complication was limited. Further research is warranted to elucidate the pathophysiology as well as help clinician coping with this notorious adverse event, advancing towards personalized medicine in oncology treatment.

\section{Conflicts of Interest}

The authors declared no conflicts of interests.

\section{Authors' Contributions}

Chau Yee Ng and Chun-Bing Chen contributed equally to this work.

\section{References}

[1] J. C. Roujeau, "Immune mechanisms in drug allergy," Allergology International, vol. 55, no. 1, pp. 27-33, 2006.

[2] L. E. French, J. T. Trent, and F. A. Kerdel, "Use of intravenous immunoglobulin in toxic epidermal necrolysis and StevensJohnson syndrome: our current understanding," International Immunopharmacology, vol. 6, no. 4, pp. 543-549, 2006.

[3] M. Mockenhaupt and E. Schopf, "Epidemiology of druginduced severe skin reactions," Seminars in Cutaneous Medicine and Surgery, vol. 15, no. 4, pp. 236-243, 1996.

[4] J. Revuz, D. Penso, J. C. Roujeau et al., "Toxic epidermal necrolysis. Clinical findings and prognosis factors in 87 patients," Archives of Dermatology, vol. 123, no. 9, pp. 1160-1165, 1987.

[5] J. C. Roujeau, J. C. Guillaume, J. P. Fabre, D. Penso, M. L. Flechet, and J. P. Girre, "Toxic epidermal necrolysis (Lyell syndrome). Incidence and drug etiology in France, 19811985," Archives of Dermatology, vol. 126, no. 1, pp. 3742, 1990.

[6] M. E. Scheulen, R. A. Hilger, C. Oberhoff et al., "Clinical phase I dose escalation and pharmacokinetic study of highdose chemotherapy with treosulfan and autologous peripheral blood stem cell transplantation in patients with advanced malignancies," Clinical Cancer Research, vol. 6, no. 11, pp. 4209-4216, 2000.

[7] I. Aydogdu, C. Ozcan, M. Harputluoglu, Y. Karincaoglu, O. Turhan, and A. Ozcanu, "Severe adverse skin reaction to chlorambucil in a patient with chronic lymphocytic leukemia," Anti-Cancer Drugs, vol. 8, no. 5, pp. 468-469, 1997.

[8] F. Pietrantonio, L. Moriconi, F. Torino, A. Romano, and A. Gargovich, "Unusual reaction to chlorambucil: a case report," Cancer Letters, vol. 54, no. 3, pp. 109-111, 1990.

[9] J. M. Newman, J. M. Rindler, W. F. Bergfeld, and J. K. Brydon, "Stevens-Johnson syndrome associated with topical nitrogen mustard therapy," Journal of the American Academy of Dermatology, vol. 36, no. 1, pp. 112-114, 1997.

[10] N. Sarma, "Stevens-Johnson syndrome and toxic epidermal necrolysis overlap due to oral temozolomide and cranial radiotherapy," American Journal of Clinical Dermatology, vol. 10, no. 4, pp. 264-267, 2009.

[11] R. Jones, M. Kirkup, S. Guglani, and K. Hopkins, "Toxic epidermal necrolysis after PCV combination chemotherapy for relapsed B-cell lymphoma," Clinical Oncology, vol. 18, no. 1, p. 90, 2006.

[12] U. Garbarini and G. P. Valassina, "Report of a case of Lyell's syndrome during a course of treatment with Natulan," Minerva Medica, vol. 64, no. 53, pp. 2775-2778, 1973.

[13] J. Guerrin and R. Michiels, "Syndrome de Lyell avee agranulocyte et thrombopenie au cours d'une chimiotherapie antimitotique," Rev Med Dijon, vol. 4, pp. 523-525, 1969. 
[14] A. Hiraki, K. Aoe, T. Murakami, T. Maeda, R. Eda, and H. Takeyama, "Stevens-Johnson syndrome induced by paclitaxel in a patient with squamous cell carcinoma of the lung: a case report," Anticancer Research, vol. 24, no. 2C, pp. 11351137, 2004.

[15] J. G. Kattan, F. S. Farhat, G. Y. Chahine et al., "Weekly docetaxel, zoledronic acid and estramustine in hormonerefractory prostate cancer (HRPC)," Investigational New Drugs, vol. 26, no. 1, pp. 75-79, 2008.

[16] C. Moisidis and V. Möbus, "Erythema multiforme major following docetaxel," Archives of Gynecology and Obstetrics, vol. 271, no. 3, pp. 268-270, 2005.

[17] Y. Sawada, K. Sugita, R. Kabashima, M. Nakamura, and Y. Tokura, "Docetaxel-induced Stevens-Johnson syndrome with regenerating epidermis composed of atypical keratinocytes," Journal of the European Academy of Dermatology and Venereology, vol. 23, no. 11, pp. 13331335, 2009.

[18] S. P. Dourakis, V. A. Sevastianos, A. Alexopoulou, M. Deutsch, and N. Stavrianeas, "Treatment side effects. Case 2. Toxic, epidermal, necrolysis-like reaction associated with docetaxel chemotherapy," Journal of Clinical Oncology, vol. 20, no. 13, pp. 3030-3032, 2002.

[19] C. H. Ohlmann, S. Kohlmorgen, D. Sahi, U. Engelmann, and A. Heidenreich, "Lethal course after chemotherapy with docetaxel. Acute liver failure with accompanying erythema multiforme major," Der Urologe, vol. 46, no. 10, pp. 14251427, 2007.

[20] C. H. Jameson and D. L. Solanki, "Stevens-Johnson syndrome associated with etoposide therapy," Cancer Treatment Reports, vol. 67, no. 11, pp. 1050-1051, 1983.

[21] L. A. Solberg Jr., M. R. Wick, and J. E. Bruckman, "Doxorubicin-enhanced skin reaction after whole-body electron-beam irradiation for leukemia cutis," Mayo Clinic Proceedings, vol. 55, no. 11, pp. 711-715, 1980.

[22] R. J. Cuthbert, J. I. Craig, and C. A. Ludlam, "Stevens-Johnson syndrome associated with methotrexate treatment for nonHodgkin's lymphoma," The Ulster Medical Journal, vol. 62, no. 1, pp. 95-97, 1993.

[23] P. J. Moe and M. Seip, "High dose methotrexate in acute lymphocytic leukemia in childhood," Acta Paediatrica Scandinavica, vol. 67, no. 3, pp. 265-268, 1978.

[24] C. H. Yang, L. J. Yang, T. H. Jaing, and H. L. Chan, “Toxic epidermal necrolysis following combination of methotrexate and trimethoprim-sulfamethoxazole," International Journal of Dermatology, vol. 39, no. 8, pp. 621-623, 2000.

[25] H. Cakesen and A. F. Oner, "Toxic epidermal necrolysis in a girl with leukemia receiving methotrexate," Indian Pediatrics, vol. 38, no. 4, p. 426, 2001.

[26] N. Stone, S. Sheerin, and S. Burge, "Toxic epidermal necrolysis and graft vs. host disease: a clinical spectrum but a diagnostic dilemma," Clinical and Experimental Dermatology, vol. 24, no. 4, pp. 260-262, 1999.

[27] A. Ozkan, H. Apak, T. Celkan, L. Yuksel, and I. Yildiz, "Toxic epidermal necrolysis after the use of high-dose cytosine arabinoside," Pediatric Dermatology, vol. 18, no. 1, pp. 3840, 2001.

[28] M. S. Figueiredo, M. Yamamoto, and J. Kerbauy, “Toxic epidermal necrolysis after the use of intermediate dose of cytosine arabinoside," Revista da Associação Médica Brasileira, vol. 44, no. 1, pp. 53-55, 1998.
[29] M. A. Angelopoulou, C. Poziopoulos, V. A. Boussiotis, F. Kontopidou, and G. A. Pangalis, "Fludarabine monophosphate in refractory B-chronic lymphocytic leukemia: maintenance may be significant to sustain response," Leukemia \& Lymphoma, vol. 21, no. 3-4, pp. 321-324, 1996.

[30] M. S. Talamonti, P. J. Catalano, D. J. Vaughn et al., "Eastern cooperative oncology group phase I trial of protracted venous infusion fluorouracil plus weekly gemcitabine with concurrent radiation therapy in patients with locally advanced pancreas cancer: a regimen with unexpected early toxicity," Journal of Clinical Oncology, vol. 18, no. 19 , pp. 3384-3389, 2000.

[31] K. R. Sommers, K. M. Kong, D. T. Bui, J. P. Fruehauf, and R. F. Holcombe, "Stevens-Johnson syndrome/toxic epidermal necrolysis in a patient receiving concurrent radiation and gemcitabine," Anti-Cancer Drugs, vol. 14, no. 8, pp. 659$662,2003$.

[32] W. Mermershtain, A. D. Cohen, I. Lazarev, M. Grunwald, and S. Ariad, "Toxic epidermal necrolysis associated with gemcitabine therapy in a patient with metastatic transitional cell carcinoma of the bladder," Journal of Chemotherapy, vol. 15, no. 5, pp. 510-511, 2003.

[33] M. A. N. Sendur and S. Kilickap, "Stevens-Johnson syndrome after treatment with capecitabine," Clinical Oncology, vol. 20, no. 2, pp. 202-203, 2008.

[34] M. S. Tallman, D. Hakimian, C. Zanzig et al., "Cladribine in the treatment of relapsed or refractory chronic lymphocytic leukemia," Journal of Clinical Oncology, vol. 13, no. 4, pp. 983-988, 1995.

[35] P. Meunier, S. Castaigne, J. N. Bastie, O. Chosidow, and S. Aractingi, "Cutaneous reactions after treatment with 2-chlorodeoxyadenosine," Acta Dermato-Venereologica, vol. 76, no. 5, pp. 385-386, 1996.

[36] P. L. Amerio and A. Tulli, "Lyell's syndrome caused by 6mercaptopurine," Giornale Italiano di Dermatolotia. Minerva Dermatologica, vol. 44, no. 10, pp. 514-516, 1969.

[37] H. Okamoto, K. Yane, T. Yamanaka, T. Fukuda, and H. Hosoi, "The usefulness of TS-1 for the treatment of head and neck cancer," Gan to Kagaku Ryoho, vol. 30, no. 8, pp. 1119-1124, 2003.

[38] C. S. Tan, R. Lim, T. C. Lim, C. W. Aw, S. W. Yeo, and S. C. Lee, "Toxic epidermal necrolysis associated with TS-1 in a patient with gastric cancer," Japanese Journal of Clinical Oncology, vol. 41, no. 5, pp. 666-668, 2011.

[39] J. Bosch-Barrera, M. Gaztañaga, J. Ceballos et al., "Toxic epidermal necrolysis related to pemetrexed and carboplatin with vitamin B12 and folic acid supplementation for advanced non-small cell lung cancer," Onkologie, vol. 32, no. 10, pp. 580-584, 2009.

[40] C. Tummino, F. Barlesi, C. Tchouhadjian et al., "Severe cutaneous toxicity after Pemetrexed as second line treatment for a refractory non small cell lung cancer," Revue des Maladies Respiratoires, vol. 24, no. 5, pp. 635-638, 2007.

[41] G. Giaccone, M. Risio, G. Bonardi, and A. Calciati, "StevensJohnson syndrome and fatal pulmonary toxicity to combination chemotherapy containing bleomycin: a case report," Tumori, vol. 72, no. 3, pp. 331-333, 1986.

[42] A. Brodsky, I. Aparici, C. Argeri, and D. Goldenberg, "Stevens-Johnson syndrome, respiratory distress and acute renal failure due to synergic bleomycin-cisplatin toxicity," Journal of Clinical Pharmacology, vol. 29, no. 9, pp. 821-823, 1989. 
[43] Y. Umebayashi, H. Enomoto, and M. Ogasawara, "Drug eruption due to peplomycin: an unusual form of StevensJohnson syndrome with pustules," The Journal of Dermatology, vol. 31, no. 10, pp. 802-805, 2004.

[44] F. E. Eyster, C. B. Wilson, and H. I. Maibach, "Mithramycin as a possible cause of toxic epidermal necrolysis (Lyell's syndrome)," California Medicine, vol. 114, no. 2, pp. 42-43, 1971.

[45] D. Purpora, M. J. Ahern, and N. Silverman, "Toxic epidermal necrolysis after mithramycin," The New England Journal of Medicine, vol. 299, no. 25, pp. 1412-1413, 1978.

[46] C. P. Castaneda, N. A. Brandenburg, R. Bwire, G. H. Burton, and J. B. Zeldis, "Erythema multiforme/Stevens-Johnson syndrome/toxic epidermal necrolysis in lenalidomide-treated patients," Journal of Clinical Oncology, vol. 27, no. 1, pp. 156-157, 2009.

[47] R. Wasch, T. Jakob, K. Technau, J. Finke, and M. Engelhardt, "Stevens-Johnson/toxic epidermal necrolysis overlap syndrome following lenalidomide treatment for multiple myeloma relapse after allogeneic transplantation," Annals of Hematology, vol. 91, no. 2, pp. 287-289, 2012.

[48] P. K. Boruah, S. Bolesta, and S. M. Shetty, "Possible lenalidomide-induced Stevens-Johnson syndrome during treatment for multiple myeloma," Pharmacotherapy, vol. 31, no. 9, p. 925, 2011.

[49] T. E. Clark, N. Edom, J. Larson, and L. J. Lindsey, “Thalomid ${ }^{\circledR}$ (Thalidomide) capsules: a review of the first 18 months of spontaneous postmarketing adverse event surveillance, including off-label prescribing," Drug Safety, vol. 24, no. 2, pp. 87-117, 2001.

[50] S. V. Rajkumar, M. A. Gertz, and T. E. Witzig, "Life-threatening toxic epidermal necrolysis with thalidomide therapy for myeloma," The New England Journal of Medicine, vol. 343, no. 13, pp. 972-973, 2000.

[51] S. B. Horowitz and A. L. Stirling, "Thalidomide-induced toxic epidermal necrolysis," Pharmacotherapy, vol. 19, no. 10, pp. 1177-1180, 1999.

[52] W. K. Eo, S. H. Kim, S. H. Cheon et al., "Toxic epidermal necrolysis following thalidomide and dexamethasone treatment for multiple myeloma: a case report," Annals of Hematology, vol. 89, no. 4, pp. 421-422, 2010.

[53] M. Colagrande, M. Di Ianni, G. Coletti et al., "Toxic epidermal necrolysis in a patient with primary myelofibrosis receiving thalidomide therapy," International Journal of Hematology, vol. 89, no. 1, pp. 76-79, 2009.

[54] A. R. Rodriguez, "L-asparaginase and toxic epidermal necrolysis," Journal of the Medical Association of Georgia, vol. 69, no. 5, pp. 355-357, 1980.

[55] Y. Honda, Y. Hattori, S. Katsura et al., "Stevens-Johnson syndrome-like erosive dermatitis possibly related to afatinib," European Journal of Dermatology, vol. 26, no. 4, pp. 413$414,2016$.

[56] J. Doesch, D. Debus, C. Meyer et al., "Afatinib-associated Stevens-Johnson syndrome in an EGFR-mutated lung cancer patient," Lung Cancer, vol. 95, pp. 35-38, 2016.

[57] M. Urosevic-Maiwald, T. Harr, L. E. French, and R. Dummer, "Stevens-Johnson syndrome and toxic epidermal necrolysis overlap in a patient receiving cetuximab and radiotherapy for head and neck cancer," International Journal of Dermatology, vol. 51, no. 7, pp. 864-867, 2012.

[58] W. L. Lin, W. C. Lin, J. Y. Yang et al., "Fatal toxic epidermal necrolysis associated with cetuximab in a patient with colon cancer," Journal of Clinical Oncology, vol. 26, no. 16, pp. 2779-2780, 2008.

[59] S. S. Lee and P. Y. Chu, "Toxic epidermal necrolysis caused by cetuximab plus minocycline in head and neck cancer," American Journal of Otolaryngology, vol. 31, no. 4, pp. 288-290, 2010.

[60] E. Liquete, S. Ali, R. Kammo et al., "Acute generalized exanthematous pustulosis induced by Erlotinib (Tarceva) with superimposed Staphylococcus aureus skin infection in a pancreatic cancer patient: a case report," Case Reports in Oncology, vol. 5, no. 2, pp. 253-259, 2012.

[61] D. M. Jackman, L. A. Cioffredi, L. Jacobs et al., "A phase I trial of high dose gefitinib for patients with leptomeningeal metastases from non-small cell lung cancer," Oncotarget, vol. 6, no. 6, pp. 4527-4536, 2015.

[62] J. J. Huang, S. X. Ma, X. Hou et al., "Toxic epidermal necrolysis related to AP (pemetrexed plus cisplatin) and gefitinib combination therapy in a patient with metastatic non-small cell lung cancer," Chinese Journal of Cancer, vol. 34, no. 2, pp. 94-98, 2015.

[63] J. Yoon, C. W. Oh, and C. Y. Kim, "Stevens-Johnson syndrome induced by vandetanib," Annals of Dermatology, vol. 23, Supplement 3, pp. S343-S345, 2011.

[64] D. Vidal, L. Puig, A. Sureda, and A. Alomar, "Sti571-induced Stevens-Johnson syndrome," British Journal of Haematology, vol. 119, no. 1, pp. 274-275, 2002.

[65] G. Severino, C. Chillotti, R. De Lisa, M. Del Zompo, and R. Ardau, "Adverse reactions during imatinib and lansoprazole treatment in gastrointestinal stromal tumors," The Annals of Pharmacotherapy, vol. 39, no. 1, pp. 162-164, 2005.

[66] K. Pavithran and M. Thomas, "Imatinib induced StevensJohnson syndrome: lack of recurrence following re-challenge with a lower dose," Indian Journal of Dermatology, Venereology and Leprology, vol. 71, no. 4, pp. 288-289, 2005.

[67] S. A. Rule, S. G. O'Brien, and L. C. Crossman, "Managing cutaneous reactions to imatinib therapy," Blood, vol. 100, no. 9, pp. 3434-3435, 2002.

[68] L. T. Hsiao, H. M. Chung, J. T. Lin et al., "Stevens-Johnson syndrome after treatment with STI571: a case report," British Journal of Haematology, vol. 117, no. 3, pp. 620622, 2002.

[69] H.-J. Hsieh, A. L. F. Chan, and S.-J. Lin, "Stevens-Johnson syndrome induced by combination of imatinib and allopurinol," Chemotherapy, vol. 55, no. 4, pp. 197-199, 2009.

[70] M. Mahapatra, P. Mishra, and R. Kumar, "Imatinib-induced Stevens-Johnson syndrome: recurrence after re-challenge with a lower dose," Annals of Hematology, vol. 86, no. 7, pp. 537-538, 2007.

[71] P. Jha, D. Himanshu, N. Jain, and A. K. Singh, "Imatinibinduced Stevens-Johnsons syndrome," BMJ Case Reports, vol. 2013, 2013.

[72] M. Schaich, K. Schakel, T. Illmer, G. Ehninger, and M. Bornhauser, "Severe epidermal necrolysis after treatment with imatinib and consecutive allogeneic hematopoietic stem cell transplantation," Annals of Hematology, vol. 82, no. 5, pp. 303-304, 2003.

[73] M. K. Choi, H. Y. Woo, J. Heo et al., "Toxic epidermal necrolysis associated with sorafenib and tosufloxacin in a patient with hepatocellular carcinoma," Annals of Dermatology, vol. 23, Supplement 3, pp. S404-S407, 2011. 
[74] B. Blanchet, B. Billemont, S. Barete et al., "Toxicity of sorafenib: clinical and molecular aspects," Expert Opinion on Drug Safety, vol. 9, no. 2, pp. 275-287, 2010.

[75] C. N. Yeh, W. H. Chung, S. C. Su et al., "Fas/Fas ligand mediates keratinocyte death in sunitinib-induced hand-foot skin reaction," The Journal of Investigative Dermatology, vol. 134, no. 11, pp. 2768-2775, 2014.

[76] E. I. Zimmerman, A. A. Gibson, S. Hu et al., "Multikinase inhibitors induce cutaneous toxicity through OAT6mediated uptake and MAP3K7-driven cell death," Cancer Research, vol. 76, no. 1, pp. 117-126, 2016.

[77] B. Fang, Y. Song, J. Ma, and R. C. Zhao, "Severe epidermal necrolysis after bortezomib treatment for multiple myeloma," Acta Haematologica, vol. 118, no. 2, pp. 65-67, 2007.

[78] B. M. Tijink, J. Buter, R. de Bree et al., "A phase I dose escalation study with anti-CD44v6 bivatuzumab mertansine in patients with incurable squamous cell carcinoma of the head and neck or esophagus," Clinical Cancer Research, vol. 12, no. 20, pp. 6064-6072, 2006.

[79] S. Lowndes, A. Darby, G. Mead, and A. Lister, "StevensJohnson syndrome after treatment with rituximab," Annals of Oncology, vol. 13, no. 12, pp. 1948-1950, 2002.

[80] D. R. Minor, R. Rodvien, and M. Kashani-Sabet, "Successful desensitization in a case of Stevens-Johnson syndrome due to vemurafenib," Melanoma Research, vol. 22, no. 5, pp. 410-411, 2012.

[81] A. A. Segura Huerta, P. Tordera, A. C. Cercos, A. L. Yuste, P. Lopez-Tendero, and G. Reynes, "Toxic epidermal necrolysis associated with interleukin-2," The Annals of Pharmacotherapy, vol. 36, no. 7-8, pp. 1171-1174, 2002.

[82] J. S. Wiener, J. A. Tucker Jr., and P. J. Walther, "Interleukin2 -induced dermatotoxicity resembling toxic epidermal necrolysis," Southern Medical Journal, vol. 85, no. 6, pp. 656-659, 1992.

[83] E. Dika, G. M. Ravaioli, and P. A. Fanti, "Cutaneous adverse effects during ipilimumab treatment for metastatic melanoma: a prospective study," European Journal of Dermatology, vol. 27, no. 3, pp. 266-270, 2017.

[84] K. L. Vivar, M. Deschaine, J. Messina et al., "Epidermal programmed cell death-ligand 1 expression in TEN associated with nivolumab therapy," Journal of Cutaneous Pathology, vol. 44, no. 4, pp. 381-384, 2017.

[85] N. Nayar, K. Briscoe, and P. Fernandez Penas, "Toxic epidermal necrolysis-like reaction with severe satellite cell necrosis associated with nivolumab in a patient with ipilimumab refractory metastatic melanoma," Journal of Immunotherapy, vol. 39, no. 3, pp. 149-152, 2016.

[86] E. Liniker, A. M. Menzies, B. Y. Kong et al., "Activity and safety of radiotherapy with anti-PD-1 drug therapy in patients with metastatic melanoma," OncoImmunology, vol. 5, no. 9, article e1214788, 2016.

[87] K. Polder, C. Wang, M. Duvic et al., "Toxic epidermal necrolysis associated with denileukin diftitox (DAB $\left.{ }_{389} \mathrm{IL}-2\right)$ administration in a patient with follicular large cell lymphoma," Leukemia \& Lymphoma, vol. 46, no. 12, pp. 1807$1811,2005$.

[88] T. J. Chen, W. H. Chung, and C. B. Chen, "Methotrexateinduced epidermal necrosis: a case series of 24 patients," Journal of the American Academy of Dermatology, vol. 77, no. 2, pp. 247-255.e2, 2017.
[89] L. E. Levine, M. M. Medenica, A. L. Lorincz, K. Soltani, B. Raab, and A. Ma, "Distinctive acral erythema occurring during therapy for severe myelogenous leukemia," Archives of Dermatology, vol. 121, no. 1, pp. 102-104, 1985.

[90] J. L. Bolognia, D. L. Cooper, and E. J. Glusac, "Toxic erythema of chemotherapy: a useful clinical term," Journal of the American Academy of Dermatology, vol. 59, no. 3, pp. 524-529, 2008.

[91] T. D. Horn, "Antineoplastic chemotherapy, sweat, and the skin," Archives of Dermatology, vol. 133, no. 7, pp. 905-906, 1997.

[92] P. Seshacharyulu, M. P. Ponnusamy, D. Haridas, M. Jain, A. K. Ganti, and S. K. Batra, "Targeting the EGFR signaling pathway in cancer therapy," Expert Opinion on Therapeutic Targets, vol. 16, no. 1, pp. 15-31, 2012.

[93] M. E. Lacouture, M. J. Anadkat, R. J. Bensadoun et al., "Clinical practice guidelines for the prevention and treatment of EGFR inhibitor-associated dermatologic toxicities," Support Care Cancer, vol. 19, no. 8, pp. 1079-1095, 2011.

[94] J. B. Macdonald, B. Macdonald, L. E. Golitz, P. LoRusso, and A. Sekulic, "Cutaneous adverse effects of targeted therapies: part I: inhibitors of the cellular membrane," Journal of the American Academy of Dermatology, vol. 72, no. 2, pp. 203218, 2015.

[95] A. M. Wnorowski, A. de Souza, A. Chachoua, and D. E. Cohen, "The management of EGFR inhibitor adverse events: a case series and treatment paradigm," International Journal of Dermatology, vol. 51, no. 2, pp. 223-232, 2012.

[96] B. J. Druker, F. Guilhot, S. G. O'Brien et al., "Five-year follow-up of patients receiving imatinib for chronic myeloid leukemia," New England Journal of Medicine, vol. 355, no. 23, pp. 2408-2417, 2006.

[97] P. G. Casali, A. Le Cesne, A. P. Velasco et al., "Imatinib failure-free survival (IFS) in patients with localized gastrointestinal stromal tumors (GIST) treated with adjuvant imatinib (IM): the EORTC/AGITG/FSG/GEIS/ISG randomized controlled phase III trial," Journal of Clinical Oncology, vol. 31, 2013.

[98] L. Valeyrie, S. Bastuji-Garin, J. Revuz et al., "Adverse cutaneous reactions to imatinib (STI571) in Philadelphia chromosome-positive leukemias: a prospective study of 54 patients," Journal of the American Academy of Dermatology, vol. 48, no. 2, pp. 201-206, 2003.

[99] M. Brouard and J. H. Saurat, "Cutaneous reactions to STI571," The New England Journal of Medicine, vol. 345, no. 8, pp. 618-619, 2001

[100] K. D. White, W. H. Chung, S. I. Hung, S. Mallal, and E. J. Phillips, "Evolving models of the immunopathogenesis of $\mathrm{T}$ cell-mediated drug allergy: the role of host, pathogens, and drug response," The Journal of Allergy and Clinical Immunology, vol. 136, no. 2, pp. 219-234, 2015.

[101] T. A. Duong, L. Valeyrie-Allanore, P. Wolkenstein, and O. Chosidow, "Severe cutaneous adverse reactions to drugs," Lancet, vol. 390, no. 10106, pp. 1996-2011, 2017.

[102] K. J. Gotink and H. M. W. Verheul, "Anti-angiogenic tyrosine kinase inhibitors: what is their mechanism of action?," Angiogenesis, vol. 13, no. 1, pp. 1-14, 2010.

[103] R. S. Ishak, S. A. Aad, A. Kyei, and F. S. Farhat, "Cutaneous manifestations of anti-angiogenic therapy in oncology: review with focus on VEGF inhibitors," Critical Reviews in Oncology/Hematology, vol. 90, no. 2, pp. 152-164, 2014. 
[104] B. Billemont, S. Barete, and O. Rixe, "Scrotal cutaneous side effects of sunitinib," The New England Journal of Medicine, vol. 359, no. 9, pp. 975-976, 2008.

[105] M. Ikeda, T. Fujita, Y. Amoh, S. Mii, K. Matsumoto, and M. Iwamura, "Stevens-Johnson syndrome induced by sorafenib for metastatic renal cell carcinoma," Urologia Internationalis, vol. 91, no. 4, pp. 482-483, 2013.

[106] M. E. Lacouture, M. Duvic, A. Hauschild et al., "Analysis of dermatologic events in vemurafenib-treated patients with melanoma," The Oncologist, vol. 18, no. 3, pp. 314-322, 2013.

[107] M. Arenbergerova, A. Fialova, P. Arenberger et al., "Severe diclofenac photoallergy in a patient treated with vemurafenib," Journal of the European Academy of Dermatology and Venereology, vol. 30, no. 4, pp. 713-715, 2016.

[108] T. Bellon, V. Lerma, O. Gonzalez-Valle, C. González Herrada, and F. J. de Abajo, "Vemurafenib-induced toxic epidermal necrolysis: possible cross-reactivity with other sulfonamide compounds," The British Journal of Dermatology, vol. 174, no. 3, pp. 621-624, 2016.

[109] G. Jeudy, S. Dalac-Rat, B. Bonniaud et al., "Successful switch to dabrafenib after vemurafenib-induced toxic epidermal necrolysis," British Journal of Dermatology, vol. 172, no. 5, pp. 1454-1455, 2015.

[110] M. E. Peterson, "Management of adverse events in patients with hormone receptor-positive breast cancer treated with everolimus: observations from a phase III clinical trial," Supportive Care in Cancer, vol. 21, no. 8, pp. 2341-2349, 2013.

[111] C. Ferté, A. Paci, M. Zizi et al., "Natural history, management and pharmacokinetics of everolimus-induced-oral ulcers: insights into compliance issues," European Journal of Cancer, vol. 47, no. 15, pp. 2249-2255, 2011.

[112] F. Martins, M. A. de Oliveira, Q. Wang et al., "A review of oral toxicity associated with mTOR inhibitor therapy in cancer patients," Oral Oncology, vol. 49, no. 4, pp. 293-298, 2013.

[113] U. Fuchs, A. Zittermann, H. K. Berthold et al., "Immunosuppressive therapy with everolimus can be associated with potentially life-threatening lingual angioedema," Transplantation, vol. 79, no. 8, pp. 981-983, 2005.

[114] S. Saw, H. Y. Lee, and Q. S. Ng, "Pembrolizumab-induced Stevens-Johnson syndrome in non-melanoma patients," European Journal of Cancer, vol. 81, pp. 237-239, 2017.

[115] N. Okiyama and S. I. Katz, "Programmed cell death 1 (PD-1) regulates the effector function of CD8 $\mathrm{T}$ cells via PD-L1 expressed on target keratinocytes," Journal of Autoimmunity, vol. 53, pp. 1-9, 2014.

[116] S. M. Goldinger, P. Stieger, B. Meier et al., "Cytotoxic cutaneous adverse drug reactions during anti-PD-1 therapy," Clinical Cancer Research, vol. 22, no. 16, pp. 4023-4029, 2016.

[117] D. B. Johnson, E. K. Wallender, D. N. Cohen et al., "Severe cutaneous and neurologic toxicity in melanoma patients during vemurafenib administration following anti-PD-1 therapy," Cancer Immunology Research, vol. 1, no. 6, pp. 373-377, 2013.

[118] (RegiSCAR), "Patients were diagnosed with SJS, SJS/TEN overlap or TEN according to the classification proposed by the European registry of severe cutaneous adverse reactions," 9 SJS.

[119] S. Bastuji-Garin, N. Fouchard, M. Bertocchi, J. C. Roujeau, J. Revuz, and P. Wolkenstein, "SCORTEN: a severity-ofillness score for toxic epidermal necrolysis," The Journal of Investigative Dermatology, vol. 115, no. 2, pp. 149-153, 2000.
[120] B. Sassolas, C. Haddad, M. Mockenhaupt et al., "ALDEN, an algorithm for assessment of drug causality in StevensJohnson syndrome and toxic epidermal necrolysis: comparison with case-control analysis," Clinical Pharmacology \& Therapeutics, vol. 88, no. 1, pp. 60-68, 2010.

[121] W. J. Pichler and J. Tilch, "The lymphocyte transformation test in the diagnosis of drug hypersensitivity," Allergy, vol. 59, no. 8, pp. 809-820, 2004.

[122] F. Pantano, M. Silletta, A. Iovieno et al., "Stevens-Johnson syndrome associated with reduced tear production complicating the use of cetuximab and panitumumab," International Journal of Colorectal Disease, vol. 24, no. 10, pp. 1247-1248, 2009. 


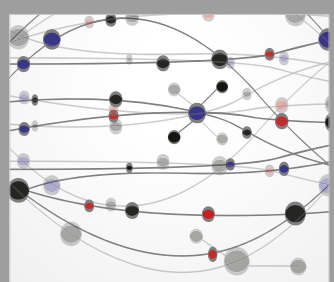

The Scientific World Journal
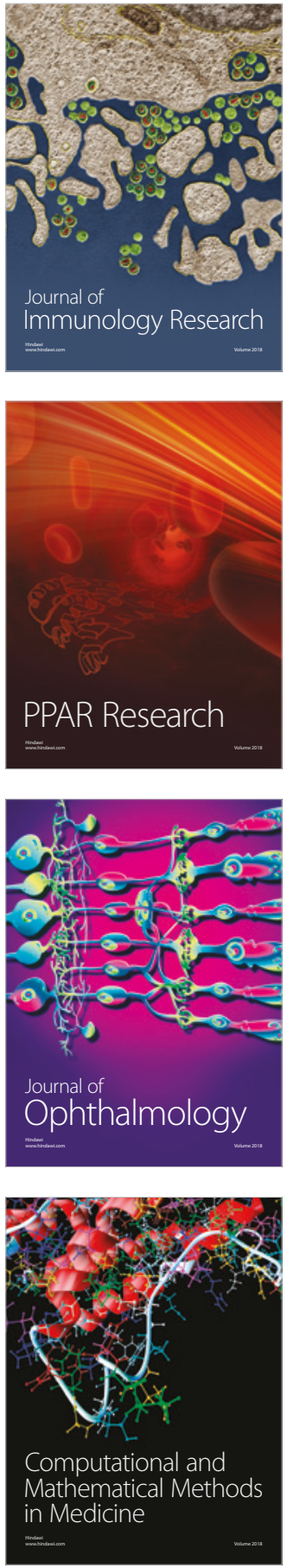

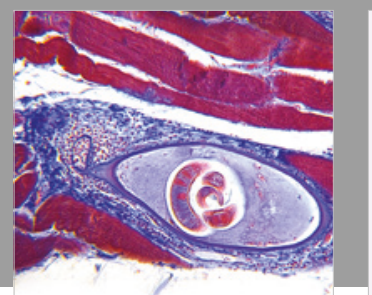

Gastroenterology Research and Practice

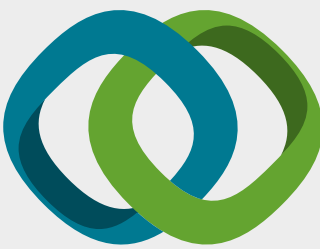

\section{Hindawi}

Submit your manuscripts at

www.hindawi.com
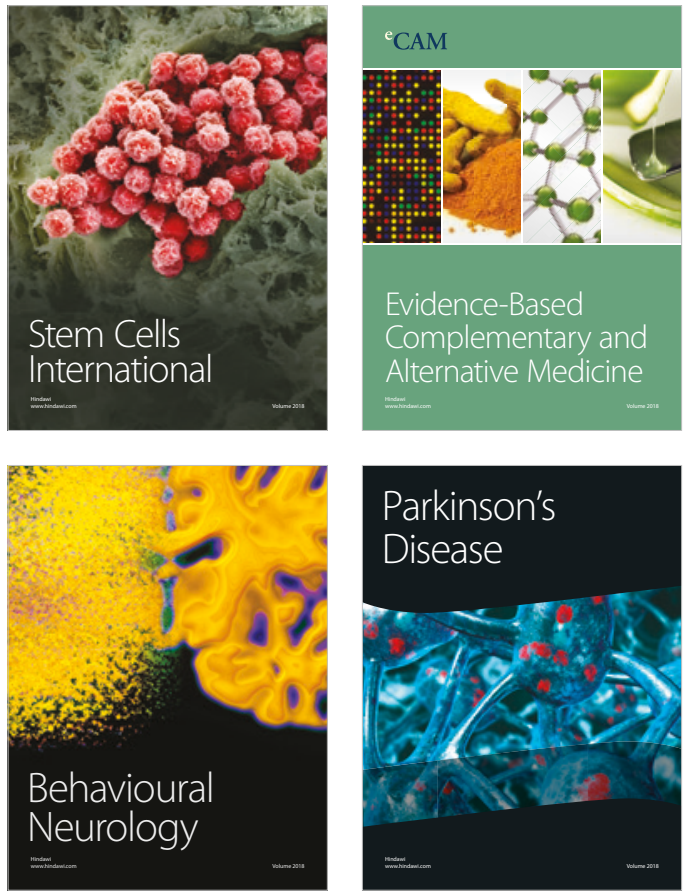

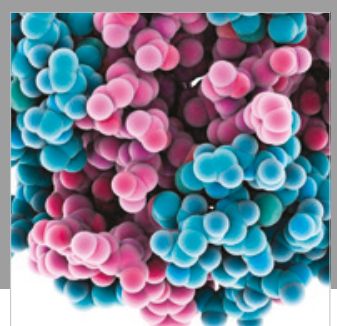

ournal of

Diabetes Research

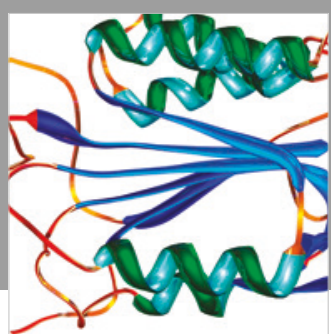

Disease Markers
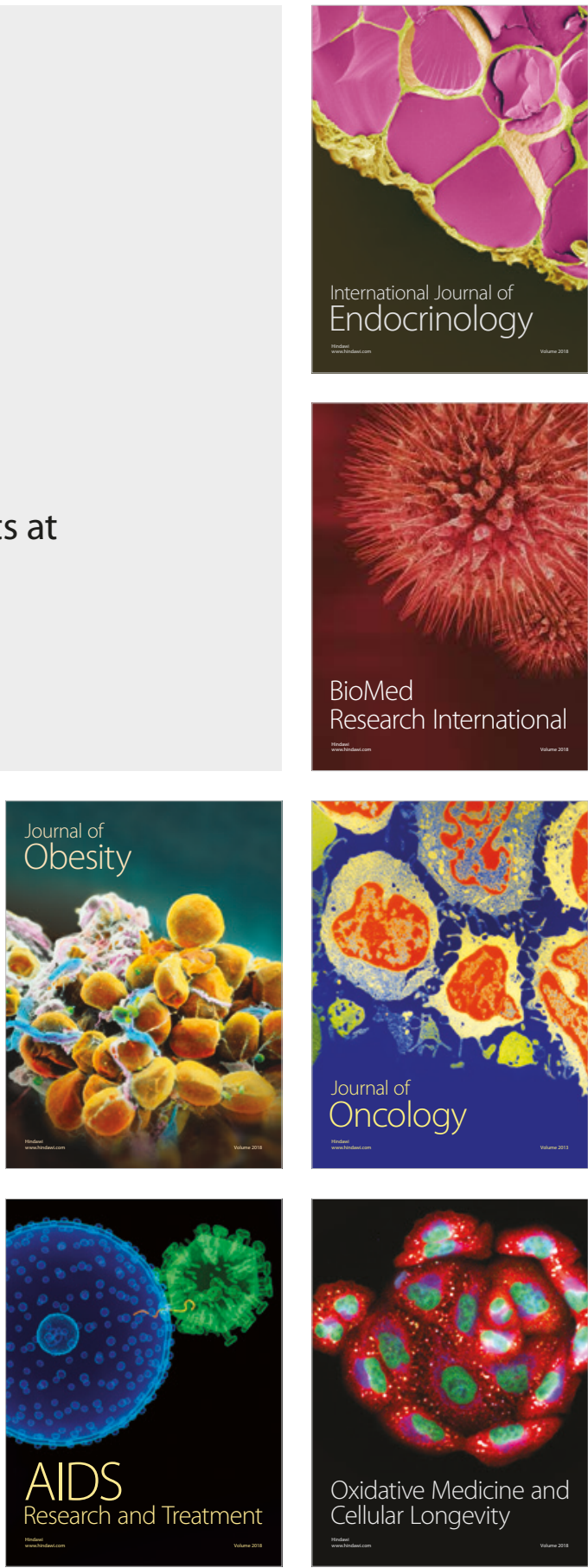